\title{
Improved Spatial Resolution of Electroencephalogram Using Tripolar Concentric Ring Electrode Sensors
}

\author{
Xiang Liu, ${ }^{1}$ Oleksandr Makeyev $\mathbb{D}^{2},{ }^{2}$ and Walter Besio $\mathbb{D}^{1}$ \\ ${ }^{1}$ Department of Electrical, Computer, and Biomedical Engineering, University of Rhode Island, 4 East Alumni Ave., Kingston, \\ RI 02881, USA \\ ${ }^{2}$ Department of Mathematics, Diné College, 1 Circle Dr., Tsaile, AZ 86556, USA
}

Correspondence should be addressed to Oleksandr Makeyev; omakeyev@dinecollege.edu

Received 22 November 2019; Accepted 29 April 2020; Published 8 June 2020

Academic Editor: Tiago H. Falk

Copyright ( $\odot 2020$ Xiang Liu et al. This is an open access article distributed under the Creative Commons Attribution License, which permits unrestricted use, distribution, and reproduction in any medium, provided the original work is properly cited.

The electroencephalogram (EEG) is broadly used for research of brain activities and diagnosis of brain diseases and disorders. Although EEG provides good temporal resolution of millisecond or less, it does not provide good spatial resolution. There are two main reasons for the poor spatial resolution: the blurring effects of the head volume conductor and poor signal-to-noise ratio. We have developed a tripolar concentric ring electrode (TCRE) Laplacian sensor and now report on computer simulations comparing spatial resolution between conventional EEG disc electrode sensors and TCRE Laplacian sensors. We also performed visual evoked stimulus experiments and acquired visual evoked potentials (VEPs) from healthy human subjects. From the simulations, we found that TCRE Laplacian sensors can provide approximately a tenfold improvement in spatial resolution and pass signals from specific volumes. Placing TCRE sensors near the brain region of interest will allow passage of the wanted signals and rejection of distant interference signals. We were also able to detect VEPs on the scalp surface and show that TCREs separated VEP sources better than conventional disc electrodes.

\section{Introduction}

Electroencephalography (EEG) is widely used in diagnosis of brain-related disorders and research. However, EEG suffers from poor spatial resolution due to the blurring effects primarily from different conductivities of the volume conductor [1]

To improve the spatial resolution, the surface Laplacian has been applied to EEG $[1,2]$. The surface Laplacian is a high-pass spatial filter, which sharpens the blurred potential distribution on the surface [2] and produces an image proportional to the cortical potentials [3].

Two approaches have been used to calculate the surface Laplacian. The global surface Laplacian approach is based on the potential interpolation on the surface [4-6]. A drawback of this approach is that building the potential interpolation equations requires a significant number of electrodes [7].

The local surface Laplacian approach approximates the surface Laplacian based on potentials from neighboring electrodes only [8]. This approach also has significant drawbacks:
(1) when the neighboring electrodes are too sparse, which is usually the case with the 10-20 system configuration, the resulting local surface Laplacian might not be a good estimation of the surface Laplacian [7], and (2) the locations where the surface Laplacian could be estimated are limited.

This paper assesses a local Laplacian that overcomes the drawback of sparse electrode distortion by employing the tripolar concentric ring electrode (TCRE; Figure 1) introduced by Besio et al. [9]. Instead of using neighboring electrodes to estimate the surface Laplacian, the three recording surfaces of a single TCRE (outer ring, middle ring, and the central disc) are used. The second drawback can also be alleviated by interpolation of the TCRE local surface Laplacian. To illustrate these points, the global surface Laplacian and local surface Laplacian are compared using a four-layer concentric inhomogeneous spherical head model [10]. This model has been selected for this study to ensure consistency with previous results of others having used it to compare Laplacian estimation methods [11]. Moreover, unlike some of the more realistic head models, it allows straightforward modeling of 


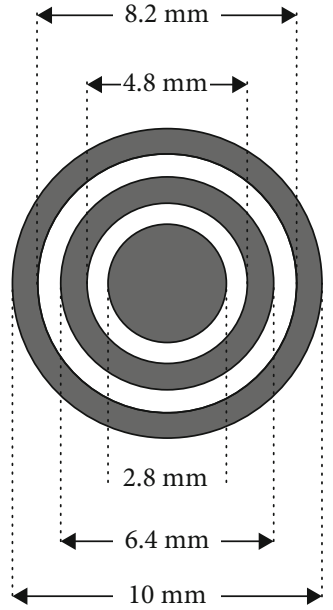

FIGURE 1: Tripolar concentric ring electrode with dimensions of its central disc, middle ring, and outer ring.

dipoles resembling visual evoked potentials, implementation of Laplacian estimation approaches, calculation of halfsensitivity volume, and application of spatial subspace decomposition. In the comparison, the global surface Laplacian estimation is based on the spherical spline interpolation method introduced by Perrin et al. [6], while the local surface Laplacian estimation is based on the TCRE Laplacian algorithm [9]. Noise is added to the simulations to make the results more realistic.

\section{Materials and Methods}

2.1. Global Surface Laplacian Estimation Based on Spherical Spline Interpolation. The spherical spline interpolation method was introduced by Perrin et al. [6]. This model approximates the head as the surface of a sphere. The equations described by Perrin et al. for the spherical spline interpolation are

$$
V(\boldsymbol{r})=c_{0}+\frac{1}{4 \pi} \sum_{i=1}^{N} c_{i} \sum_{n=1}^{\infty} \frac{2 n+1}{n^{m}(n+1)^{m}} \mathbf{p}_{n}\left(\cos \left(\boldsymbol{r}, \boldsymbol{r}_{i}\right)\right),
$$

where $N$ is the number of electrodes, $m$ is the order of the spline interpolation ( $m=3$ for this study), $r$ is the vector of the location where the potential is interpolated, $\boldsymbol{r}_{i}$ is the vector of the location of the $i^{\text {th }}$ electrode, $\mathbf{p}_{n}$ is the $n^{\text {th }}$ degree Legendre polynomial. With $n$ increasing in (1) as part of the sum, in Perrin et al. [6], $\mathbf{p}_{n}$ was "computed via the recurrence relation" and "the sum of the first 7 terms of the series"

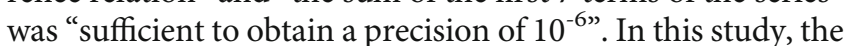
maximum value of $n$ was increased to 60 to further improve the precision. The parameters vector $C$ is the solution of equations (2) and (3):

$$
\begin{aligned}
G C+T c_{0} & =Z, \\
T^{\prime} C & =0,
\end{aligned}
$$

where $T^{\prime}=(1,1, \cdots, 1), C^{\prime}=\left(c_{1}, c_{2}, \cdots, c_{N}\right), Z^{\prime}=\left(z_{1}, z_{2}, \cdots\right.$, $\left.z_{N}\right), G=\left(g_{i j}\right)=\left(g\left(\cos \left(\boldsymbol{r}, \boldsymbol{r}_{i}\right)\right)\right)$, and $g(x)=1 / 4 \pi \sum_{\mathrm{n}=1}^{\infty}(2 n+1$ $\left./ n^{m}(n+1)^{m}\right) \mathbf{p}_{\mathbf{n}}(x)$. The surface Laplacian operator in the spherical coordinate system is defined as

$$
\Delta_{\text {surf }}=\frac{1}{r^{2} \sin \theta} \frac{\partial}{\partial \theta}\left(\sin \theta \frac{\partial}{\partial \theta}\right)+\frac{1}{r^{2} \sin ^{2} \theta} \frac{\partial^{2}}{\partial \phi^{2}} .
$$

Applying the operator from equation (4) to equation (1) produces the surface Laplacian of the spherical interpolation:

$$
\Delta_{\text {surf }} V(\boldsymbol{r})=-\frac{1}{4 \pi r^{2}} \sum_{i=1}^{N} c_{i} \sum_{n=1}^{\infty} \frac{2 n+1}{n^{m-1}(n+1)^{m-1}} \mathbf{p}_{\mathbf{n}}\left(\cos \left(\boldsymbol{r}, \boldsymbol{r}_{i}\right)\right) .
$$

We used a truncated singular value decomposition method to solve the inverse problem of the ill-posed matrix in equations (2) and (3) [12].

2.2. Local Surface Laplacian Estimation Based on Tripolar Concentric Ring Electrode. Based on the 2-dimensional Taylor expansion of the potential on the surface Laplacian nine-point locations, the tripolar Laplacian is given by the combination of the potentials from the three recording surfaces of the TCRE [9]:

$$
\mathrm{SL}=-\frac{16\left(V_{m}-V_{d}\right)-\left(V_{o}-V_{d}\right)}{3 R^{2}} .
$$

In equation (6), SL denotes the surface Laplacian, $V_{d}$ denotes the potential from the central disc, $V_{m}$ denotes the potential from the middle ring, $V_{o}$ denotes the potential from the outer ring, and $R$ is the radius of the middle ring. As $R$ changes, the size of the sensor changes, and the spatial resolution also varies with it.

2.3. The Four-Layer Spherical Head Model and the Analytical Surface Laplacian. In our simulations, we used a four-layer concentric inhomogeneous spherical model [10] to represent the human head (Figure 2). Current dipoles, described later, are employed to model the brain activity.

The potential on the surface of the model due to a current dipole located at the $z$-axis in the brain is given by the following equations [10]:

$$
V_{x}=\frac{P_{x} \cos \phi}{4 \pi \sigma_{4} R^{2}} \sum_{n=1}^{\infty} \frac{(2 n+1)^{4} f^{n-1}(c d)^{2 n+1} P_{n}^{1}(\cos \theta)}{n \Gamma},
$$

for the $x$-direction component of the dipole,

$$
V_{y}=\frac{P_{y} \sin \phi}{4 \pi \sigma_{4} R^{2}} \sum_{n=1}^{\infty} \frac{(2 n+1)^{4} f^{n-1}(c d)^{2 n+1} P_{n}^{1}(\cos \theta)}{n \Gamma},
$$




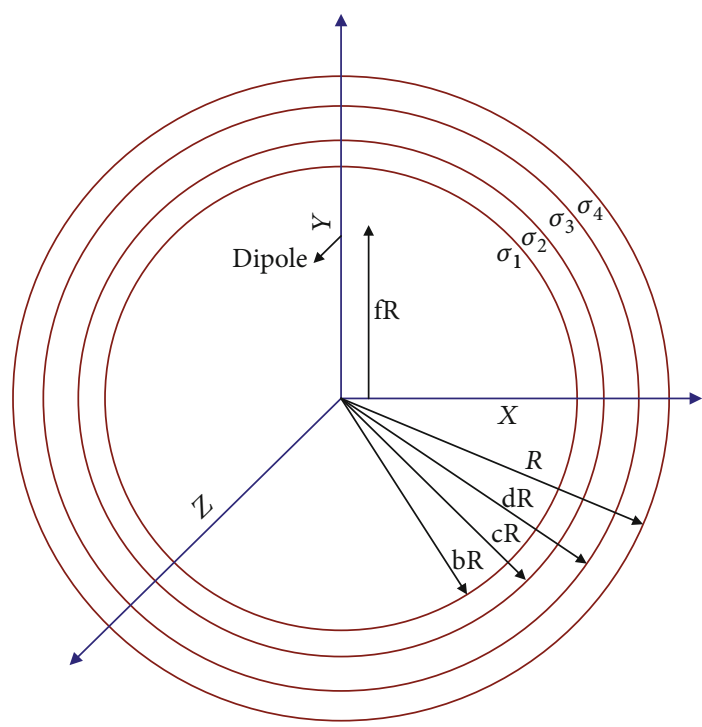

Figure 2: Four-layer concentric inhomogeneous spherical head model with the radii of the layers equal to $R=8.8 \mathrm{~cm}, \mathrm{dR}=8.5 \mathrm{~cm}$, $\mathrm{cR}=8.1 \mathrm{~cm}$, and $\mathrm{bR}=7.9 \mathrm{~cm}$ and the conductivities of the layers equal to $\sigma_{1}=3.3 \times 10^{-3}, \sigma_{2}=10.0 \times 10^{-3}, \sigma_{3}=4.2 \times 10^{-5}$, and $\sigma_{4}=$ $3.3 \times 10^{-3} \mathrm{~S} / \mathrm{cm}$, from inside to outside, respectively.

for the $y$-direction component of the dipole, and

$$
V_{z}=\frac{P_{z}}{4 \pi \sigma_{4} R^{2}} \sum_{n=1}^{\infty} \frac{(2 n+1)^{4} f^{n-1}(c d)^{2 n+1} P_{n}^{1}(\cos \theta)}{n \Gamma},
$$

for the $z$-direction component of the dipole, where

$$
\begin{aligned}
\Gamma= & d^{2 n+1}\left\{b^{2 n+1} n\left(k_{1}-1\right)\left(k_{2}-1\right)(n+1)\right. \\
& \left.+C^{2 n+1}\left(k_{1} n+n+1\right)\left(k_{2} n+n+1\right)\right\} \\
& \times\left\{\left(k_{3} n+n+1\right)+(n+1)\left(k_{3}-1\right) d^{2 n+1}\right\} \\
& +(n+1) c^{2 n+1}\left\{b^{2 n+1}\left(k_{1}-1\right)\left(k_{2} n+k_{2}+n\right)\right. \\
& \left.+c^{2 n+1}\left(k_{1} n+n+1\right)\left(k_{2}-1\right)\right\} \\
& \times\left\{n\left(k_{3}-1\right)+\left(k_{3} n+k_{3}+n\right) d^{2 n+1}\right\}, \\
k_{1}= & \frac{\sigma_{1}}{\sigma_{2}} \\
k_{2}= & \frac{\sigma_{2}}{\sigma_{3}} \\
k_{3}= & \frac{\sigma_{3}}{\sigma_{4}} .
\end{aligned}
$$

Applying the surface Laplacian operator equation (4) to equations (7), (8), and (9), the analytical surface Laplacian is given by

$$
\begin{aligned}
\Delta_{\text {surf }} V_{x}= & \frac{P_{x} \cos \phi}{4 \pi \sigma_{4} R^{2}} \sum_{n=1}^{\infty} \frac{1}{n \Gamma} \times\left\{(2 n+1)^{4} f^{n-1}(c d)^{2 n+1}\right. \\
& \left.\cdot\left(-\frac{P_{n}^{1}(\cos \theta)}{R^{2} \sin ^{2} \theta}+\Delta_{\text {surf }} P_{n}^{1}(\cos \theta)\right)\right\},
\end{aligned}
$$

$$
\begin{aligned}
\Delta_{\text {surf }} V_{y}= & \frac{P_{y} \sin \phi}{4 \pi \sigma_{4} R^{2}} \sum_{n=1}^{\infty} \frac{1}{n \Gamma} \times\left\{(2 n+1)^{4} f^{n-1}(c d)^{2 n+1}\right. \\
& \left.\cdot\left(-\frac{P_{n}^{1}(\cos \theta)}{R^{2} \sin ^{2} \theta}+\Delta_{\text {surf }} P_{n}^{1}(\cos \theta)\right)\right\}, \\
\Delta_{\text {surf }} V_{z}= & \frac{P_{z}}{4 \pi \sigma_{4} R^{2}} \sum_{n=1}^{\infty} \frac{1}{n \Gamma} \\
& \times\left\{(2 n+1)^{4} f^{n-1}(c d)^{2 n+1} \Delta_{\text {surf }} P_{n}^{1}(\cos \theta)\right\},
\end{aligned}
$$

where

$$
\begin{aligned}
\Delta_{\text {surf }} P_{n}^{1}(\cos \theta)= & \frac{1}{R^{2} \sin ^{3} \theta}\left\{P _ { n } ( \operatorname { c o s } \theta ) \left(n(n+1)^{2} \cos \theta \sin ^{2} \theta\right.\right. \\
& -(n+1) \cos \theta)+P_{n+1}(\cos \theta)((n+1) \\
& \left.\left.-n(n+1) \sin ^{2} \theta\right)\right\}, \\
\Delta_{\text {surf }} P_{n}(\cos \theta)= & -\frac{n(n+1)}{R^{2}} P_{n}(\cos \theta) .
\end{aligned}
$$

By rotating the coordinate system, the analytical potential and surface Laplacian imposed by a dipole at an arbitrary brain location area can be computed according to equations (7)-(9) and (11)-(13).

2.4. Sensitivity Distribution of Conventional Electrodes and TCREs Based on Half-Sensitivity Volume. The sensitivity distribution of an electrode is directly related to its spatial resolution. In this comparison, the lead field was used to calculate the sensitivity distribution. The lead field is the current density distribution in the volume conductor generated by feeding current to electrode pairs [13]. We also employed the concept of half-sensitivity volume (HSV), which is defined as the volume where the measured sensitivity is at least half of the maximum sensitivity [13], to quantize the sensitivity distribution for the electrodes.

2.5. Sensitivity Comparison of Conventional Electrodes and TCREs Based on Spatial Subspace Decomposition Method. Common spatial subspace decomposition (CSSD), which helps to retrieve signal components specific to one condition from complex EEG background, was developed to separate specific brain activities from the background [14]. Since EEG is considered to have spatial resolution of 3.0 to $4.0 \mathrm{~cm}$ [15-17], we tested at a higher spatial resolution for comparison. In our simulation, an 8 by 8 simulated electrode array was placed on the scalp above the visual cortex area with a $1.0 \mathrm{~cm}$ center-to-center distance between electrodes to maximize the spatial resolution. Potential integration was performed separately and independently for each electrode to eliminate mutual influence of neighboring electrodes. A simulated signal dipole with eccentricity of 0.9 was placed under the electrode array. Two simulated noise dipoles with an eccentricity of 0.75 were concurrently activated with the signal dipole under the array as background brain activity. In the simulation, we first calculated the simulated background by setting the magnitude of the signal dipole to zero. Then, 
TABLE 1: Locations and moments of the ten dipoles for modeling brain activities.

\begin{tabular}{lcccc}
\hline Dipole number & $X(\mathrm{~cm})$ & $Y(\mathrm{~cm})$ & $Z(\mathrm{~cm})$ & $\begin{array}{c}\text { Moment (R, radial; T, } \\
\text { tangential; U, unit; and } \\
\text { D, dipole) }\end{array}$ \\
\hline 1 & 4.3 & -5.3 & 4 & RUD \\
2 & 6 & -3 & 4 & RUD \\
3 & 5 & -4.6 & 4.1 & RUD \\
4 & -2.3 & -4.4 & 6 & RUD \\
5 & -2.2 & 4.6 & 6 & RUD \\
6 & 4.3 & -5.3 & 4 & TUD \\
7 & 6 & -3 & 4 & TUD \\
8 & 5 & -4.6 & 4.1 & TUD \\
9 & -2.3 & -4.4 & 6 & TUD \\
10 & -2.2 & 4.6 & 6 & TUD \\
\hline
\end{tabular}

we calculated the simulated visual evoked potential (VEP) combined with background. Finally, the CSSD was applied to the simulated data to extract the VEP. The simulated TCRE EEG (tEEG) VEP from the TCRE was calculated for comparison. Another simulation with only the signal dipole was also conducted to compare the power distribution of the simulated disc potential and tripolar Laplacian. In all of the simulations, potentials on the disc electrodes were calculated from the conventional disc electrodes that had the same diameter as the outer ring of the TCRE, $1.0 \mathrm{~cm}$.

2.6. Comparison of Global Spline Surface Laplacian and Local TCRE Surface Laplacian with Computer Simulation. To model the activities of the brain cortex area, ten dipoles with an eccentricity around 0.89 were used one at a time (Table 1 ). The locations of the dipoles were modeled in the visual cortex area of the brain to compare the simulation results to those of actual VEP recording experiments. The moments of the first five dipoles had a radial direction, and the remaining five dipoles were at the same locations, but with a tangential direction.

Since an electrode shunts the scalp area under it, to simulate the potential on the recording surfaces of the TCREs and conventional disc electrodes, we averaged a number of "sampling points" uniformly distributed on the surface of the electrode. To determine the number of sampling points needed for stable calculations, we incrementally increased their density and compared the averaged potential until the difference in potential due to adding more points was less than $0.1 \%$. The order of magnitude of that number was in the thousands of sampling points per electrode. We used the same density of sampling points for each of the recording surfaces of the TCREs and the same sampling points for each TCRE. A similar procedure was used for the disc electrodes. In the simulation, TCREs were given the same dimensions as shown in Figure 1, and conventional disc electrodes were simulated with the same diameter as the outer ring of the TCREs, $1.0 \mathrm{~cm}$.

The global spline surface Laplacian and the local TCRE surface Laplacian were calculated at the locations of the elec- trodes and then compared to the analytical surface Laplacian using the correlation coefficient.

2.7. Statistical Analysis. All the statistical analysis was performed using Design-Expert software (Stat-Ease Inc., Minneapolis, MN, USA). Full factorial design of analysis of variance (ANOVA) was used with four categorical factors [18]. The first factor (A) was the type of the electrode presented at two levels corresponding to conventional disc electrodes and tripolar concentric ring electrodes. The second factor (B) was the number of electrodes presented at four levels corresponding to $19,32,64$, and 128 electrodes. The 19 electrodes were placed at the standard 10-20 system while 32 , 64, and 128 electrode locations were selected from the 5-5 system [19]. The third factor (C) was the presence and type of noise presented at four levels corresponding to no noise, presence of white Gaussian noise (WGN) at 20\% standard deviation ratio of the WGN to the potential [20], presence of a deep noise dipole with an eccentricity of around 0.85 (simulating brain activity not considered to be the brain source of interest), and presence of both WGN and the noise dipole. Finally, the fourth factor (D) was the dipole location presented at ten levels corresponding to 10 signal dipole locations from Table 1. The response variable was the correlation coefficient of the simulated surface Laplacian and the analytical surface Laplacian calculated for each of the $2 * 4 * 4 *$ $10=320$ combinations of levels of four factors. The full factorial design of our study is presented in Table 2.

2.8. Visual Evoked Surface Potential and Laplacian Recording Experiment. In this experiment, the scalp was prepared with the mild abrasive NuPrep (Natus Medical West Warwick RI). Next, recording electrodes with approximately $0.2 \mathrm{~cm}$ of Ten 20 paste (for skin-to-electrode impedance matching and to hold the electrodes in place) were placed over the visual cortex. Finally, reference and ground electrodes were placed on the forehead between the eyes in an identical manner. Signals from the outer ring of the TCREs were used to emulate the disc electrodes. Synchrony between these two signals has been demonstrated in time domain using crosscorrelation in phantom and human data $(r \geq 0.99)$ [21] as well as in frequency domain using coherence in human data $(C \geq 0.98)$ [22]. Both of the results strongly suggesting equivalency of signals from the outer ring of the TCRE, and signals from conventional disc electrodes were later confirmed on a more comprehensive human dataset [23]. A flashing LED array, PS60/LED, and Comet AS40 (Natus Medical, West Warwick, RI) were used to activate the visual cortex, similar to the computer model, of the human brain and record the EEG. The visual stimulus was expected to generate a signal source in the visual cortex similar to the dipoles we placed in the computer simulation. The signals were filtered (1$70 \mathrm{~Hz})$ and digitized $(200 \mathrm{~S} / \mathrm{s})$. Due to the limit of the hardware, only 15 channels were available in the experiments. To keep the electrodes at a similar density as we used in the simulation, all 15 electrodes were placed over the visual cortex area from the standard 10-5 system. The locations of the electrodes are listed in Table 3. The frequency of the PS60/LED was $2 \mathrm{~Hz}$. The subjects $(n=6)$ were seated in a 
TABLE 2: Full factorial design of analysis of variance and obtained response variable.

\begin{tabular}{|c|c|c|c|c|}
\hline $\begin{array}{l}\text { Group averages for } 10 \text { levels of } \\
\text { factor D (signal dipole location) }\end{array}$ & $\begin{array}{l}\text { A: type of } \\
\text { the electrode }\end{array}$ & $\begin{array}{l}\text { tegorical factors } \\
\text { B: number } \\
\text { of electrodes }\end{array}$ & $\begin{array}{l}\text { C: presence and } \\
\text { type of noise }\end{array}$ & $\begin{array}{l}\text { Correlation between the simulated } \\
\text { and the analytical surface Laplacians } \\
\text { (mean } \pm \text { standard deviation) }\end{array}$ \\
\hline 1 & Conventional disc & 19 & No noise & $0.5882 \pm 0.1581$ \\
\hline 2 & TCRE & 19 & No noise & $0.9908 \pm 0.0196$ \\
\hline 3 & Conventional disc & 32 & No noise & $0.6669 \pm 0.1693$ \\
\hline 4 & TCRE & 32 & No noise & $0.9823 \pm 0.0406$ \\
\hline 5 & Conventional disc & 64 & No noise & $0.8242 \pm 0.1141$ \\
\hline 6 & TCRE & 64 & No noise & $0.9937 \pm 0.0073$ \\
\hline 7 & Conventional disc & 128 & No noise & $0.8885 \pm 0.0989$ \\
\hline 8 & TCRE & 128 & No noise & $0.9737 \pm 0.0311$ \\
\hline 9 & Conventional disc & 19 & WGN & $0.4801 \pm 0.2041$ \\
\hline 10 & TCRE & 19 & WGN & $0.9649 \pm 0.0104$ \\
\hline 11 & Conventional disc & 32 & WGN & $0.6035 \pm 0.1138$ \\
\hline 12 & TCRE & 32 & WGN & $0.9634 \pm 0.0074$ \\
\hline 13 & Conventional disc & 64 & WGN & $0.7095 \pm 0.0139$ \\
\hline 14 & TCRE & 64 & WGN & $0.9619 \pm 0.0411$ \\
\hline 15 & Conventional disc & 128 & WGN & $0.7515 \pm 0.0783$ \\
\hline 16 & TCRE & 128 & WGN & $0.9633 \pm 0.0050$ \\
\hline 17 & Conventional disc & 19 & Noise dipole & $0.4662 \pm 0.2787$ \\
\hline 18 & TCRE & 19 & Noise dipole & $0.8846 \pm 0.1186$ \\
\hline 19 & Conventional disc & 32 & Noise dipole & $0.6199 \pm 0.2052$ \\
\hline 20 & TCRE & 32 & Noise dipole & $0.9236 \pm 0.0877$ \\
\hline 21 & Conventional disc & 64 & Noise dipole & $0.7950 \pm 0.1177$ \\
\hline 22 & TCRE & 64 & Noise dipole & $0.9549 \pm 0.0424$ \\
\hline 23 & Conventional disc & 128 & Noise dipole & $0.9082 \pm 0.0904$ \\
\hline 24 & TCRE & 128 & Noise dipole & $0.9877 \pm 0.1334$ \\
\hline 25 & Conventional disc & 19 & WGN+dipole & $0.4752 \pm 0.0224$ \\
\hline 26 & TCRE & 19 & WGN+dipole & $0.9480 \pm 0.1864$ \\
\hline 27 & Conventional disc & 32 & WGN+dipole & $0.6780 \pm 0.0738$ \\
\hline 28 & TCRE & 32 & WGN+dipole & $0.9390 \pm 0.0376$ \\
\hline 29 & Conventional disc & 64 & WGN+dipole & $0.7329 \pm 0.0156$ \\
\hline 30 & TCRE & 64 & WGN+dipole & $0.9551 \pm 0.0611$ \\
\hline 31 & Conventional disc & 128 & WGN+dipole & $0.7614 \pm 0.0881$ \\
\hline 32 & TCRE & 128 & WGN+dipole & $0.9580 \pm 0.0097$ \\
\hline
\end{tabular}

comfortable chair with their eyes approximately $4.0 \mathrm{~cm}$ from the photic stimulator. For each subject, we recorded about two-and-a-half minutes of EEG signals. There was approximately 30 seconds of baseline EEG, with no photic stimulation, and then approximately two minutes of photic stimulation.

The photic trigger signal was also recorded to synchronize epochs during ensemble averaging. The analysis of recorded EEG signals depended on the type of signals recorded. For the EEG from the outer ring of the TCREs, the spline interpolation and surface Laplacian methods discussed above were applied to calculate the spline surface
Laplacian and map them to the surface of the spherical head model over the visual cortex area. For the TCRE EEG surface Laplacian, we simply applied the interpolation algorithm to map the recorded Laplacian values to the corresponding surface.

\section{Results}

3.1. Sensitivity Distribution of Conventional Electrodes and TCREs Based on Half-Sensitivity Volume. Figure 3 shows the simulated HSV of a pair of conventional disc electrodes and a TCRE. In the HSV computer simulation, a pair of disc 
TABLe 3: Electrode locations in the VEP experiments.

\begin{tabular}{lccc}
\hline Electrode location & $X(\mathrm{~cm})$ & $Y(\mathrm{~cm})$ & $Z(\mathrm{~cm})$ \\
\hline CP5 & -7.885 & -2.974 & 2.499 \\
P3 & -4.990 & -5.958 & 4.127 \\
Pz & 0.000 & -6.283 & 6.151 \\
P4 & 4.981 & -5.958 & 4.127 \\
CP6 & 7.885 & -2.974 & 2.499 \\
P5 & -6.521 & -5.588 & 1.874 \\
P6 & 6.521 & -5.588 & 1.883 \\
P7 & -7.075 & -5.157 & -0.774 \\
PO7 & -5.139 & -7.101 & -0.616 \\
PO3h & -2.526 & -8.008 & 2.622 \\
POz & 0.000 & -8.175 & 3.238 \\
PO4h & 2.517 & -8.008 & 2.622 \\
P8 & 7.075 & -5.166 & -0.774 \\
O1 & -2.702 & -8.351 & -0.414 \\
O2 & 2.702 & -8.351 & -0.414 \\
\hline
\end{tabular}

electrodes was placed on the spherical surface separated by 90 degrees. This separation angle was selected based on where the reference and signal electrode placements were in physical experiments. In our physical human VEP experiments, the separation angle, from the forehead to the visual cortex, was more like 180 degrees, rather than just 90 degrees; however, the larger angle would not affect the results. A single TCRE was utilized since it can be seen as a combination of two pairs of electrodes at a single location: the outer ring minus the disc and the middle ring minus the disc. Simulated potentials on the electrodes were calculated from a unit dipole located in the inner sphere of the brain. After the potentials were calculated, the dipole was moved. This procedure was repeated until the HSV volume could be determined. The simulation shows that the HSV of the disc electrode is 9.6 times greater than the HSV of TCREs.

\subsection{Sensitivity Comparison of Conventional Electrodes and} TCREs Based on Spatial Subspace Decomposition Method. The 64 extracted signals from the $8 \times 8$ arrays of TCREs and disc electrodes were normalized separately. The average power of the 64 normalized disc potentials was equal to $0.44 \pm 0.31$ while the average power of the 64 normalized tripolar Laplacians was equal to $0.23 \pm 0.24$ (mean \pm standard deviation). These results indicate that the distribution of the power of the tripolar Laplacian is more focused on a smaller number of TCREs, while the power of the disc potential tends to be distributed over a larger number of disc electrodes.

Figure 4 shows the simulated normalized VEP from a location near the center of the 64-electrode array. The $x$-axis is the distance from the electrodes to the signal dipole, the $y$-axis is the normalized magnitude of the signal calculated at each electrode of the $8 \times 8$ array, " $*$ " denotes the disc electrode, "o" denotes the TCRE, and "+" denotes the analytical Laplacian. From Figure 4, as the distance increases between the electrode and the dipole source, the magnitude of the recorded signal on TCREs attenuates much quicker than that recorded on the disc electrodes. In other words, the VEP power was mainly distributed on just a few close TCREs, while it was distributed over a wider area of the conventional disc electrode array. It can also be seen that the TCRE Laplacian is very similar to the analytical Laplacian.

\subsection{Comparison of Global Spline Surface Laplacian and Local} TCRE Surface Laplacian with Computer Simulation. Correlation coefficient data obtained in this simulation for $320 \mathrm{com}$ binations of factor levels is presented in Table 2 averaged for ten dipole locations.

The effect of factors A, B, C, and D on the correlation coefficient was assessed along with the effect of all possible two- and three-factor interactions. The effect of the fourfactor interaction $A B C D$ could not be evaluated. The ANOVA results suggest that all the factors and all of the assessed interactions have statistically significant effects in the model (d.f. $=238, F=17.6, p<0.0001$ ) for the optimal power transformation of 2.81 determined using the BoxCox procedure [18]. The effects of the main factors were A (d.f. $=1, F=2736.5, p<0.0001$ ), B (d.f. $=3, F=120.1, p<$ 0.0001 ), C (d.f. $=3, F=34.7, p<0.0001$ ), and D (d.f. $=9$, $F=10.3, p<0.0001)$.

3.4. Visual Evoked Surface Laplacian Comparison Experiments. From Figure 5, we can see that the TCRE Laplacian sensors were able to separate VEP sources. In panel (a), the spline Laplacian map from the 15 disc electrode signals at $95 \mathrm{~ms}$ in panel (c), in the top central area there is a red and orange area (designated with an arrow). In the same area of panel (b), from $110 \mathrm{~ms}$ in panel (d), we can see the TCRE Laplacian sensor map from the 15 TCRE signals which shows that there were two distinct sources (shown by arrow). Panels (c) and (d) show the normalized grand-averaged EEG and tEEG VEPs used to build the maps in panels (a) and (b), respectively. From panel (c), it can be seen that many of the traces are similar while this is not the case in panel (d) from the TCREs. From panels (c) and (d), we can see that there is a positive wave at approximately 50 to $110 \mathrm{~ms}$ and 105 to $115 \mathrm{~ms}$, respectively, after the photic stimulation pulse.

\section{Discussion}

We conducted multiple computer simulations and acquired real signals to compare spatial sensitivity between disc electrode and TCRE sensors. The sensitivity comparison of the disc electrode spline Laplacian and tripolar Laplacian based on HSV shows that the tripolar Laplacian is more sensitive than the disc electrode spline Laplacian. The HSV for the tripolar Laplacian is nearly 10 times smaller than the disc electrode spline Laplacian HSV (Figure 3). These results show that the tripolar Laplacian records signals from a local volume compared to two broad volumes for the disc electrode spline Laplacian.

We also used the CSSD method and showed that TCRE sensors are more focused on local potentials. This can be explained in terms of obtained HSV results. The TCRE sensors are sensitive to local sources so only the sensors that 


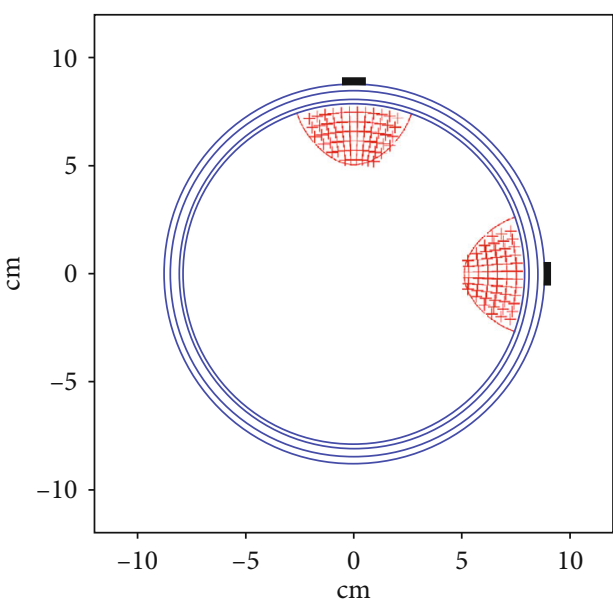

(a)

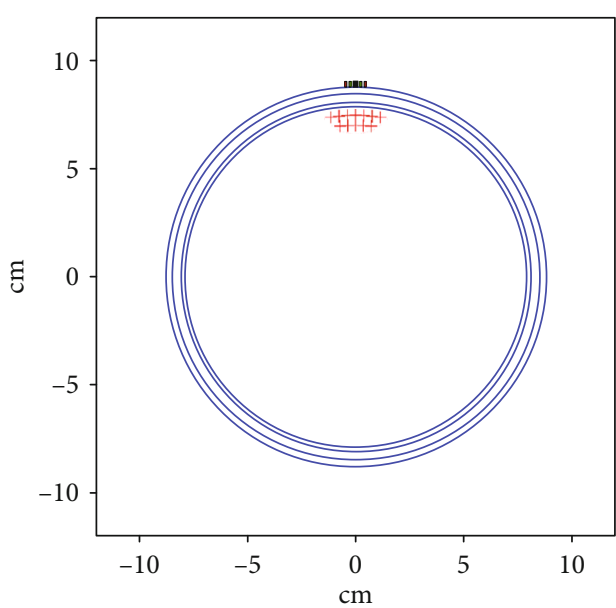

(b)

FIgure 3: The red (hashed) lines show the HSV of conventional disc electrodes (a) and TCREs (b).

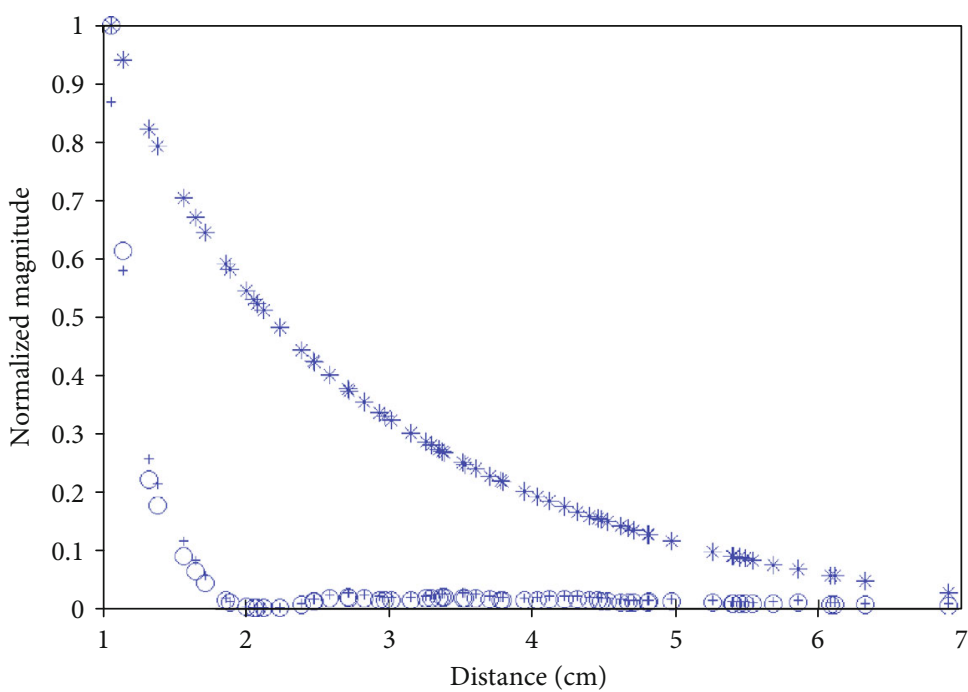

OTCRE signal

* Disc eletrode signal

+ Analytical surface Laplacian

FIGURE 4: A comparison, at a location near the center of the $8 \times 8$ array, of the calculated spline Laplacian “*," TCRE Laplacian "o," and the analytical Laplacian, the gold standard "+." The spline Laplacian was calculated from the disc electrode potentials. The disc electrode potentials were calculated from a uniform density of points over the outer ring of the $1.0 \mathrm{~cm}$ diameter TCRE similar to the way the outer ring of the TCRE was used as an emulation of the conventional disc electrode in real visual evoked potential experiments based on their equivalency [21-23]. The tripolar Laplacian matches the analytical Laplacian trace very well.

are close to the sources (whether they are signal or noise sources) will correspond to high power. At the same time, conventional disc electrodes, which have a nearly 10 -fold larger HSV, record signals from a much larger volume therefore providing less discrimination between source locations. This relative lack of discrimination for conventional disc electrodes suggests that we can place TCRE sensors closer together (i.e., at higher spatial resolution than disc electrodes) and still detect independent sources.

ANOVA results for comparing the global spline surface Laplacian to the local TCRE surface Laplacian show statistical significance of the effect of all four categorical factors included in this study. While it was important to confirm that the quality of Laplacian estimation increases with an increase in the number of electrodes (factor B), decreases in the presence of the noise (factor $C$ ), and is affected by the signal dipole location (factor D), the most important result is that, for the case of the factor A, the local TCRE Laplacian is significantly better than the global spline Laplacian at approximating the analytic Laplacian.

A potential limitation of the current full factorial design is that we could not assess the effect of interaction of all four factors. Without replications, including this interaction into the model makes it overspecified with all the degrees of freedom being in the model and none assigned to the residual (error). On the other hand, adding replications to the design 


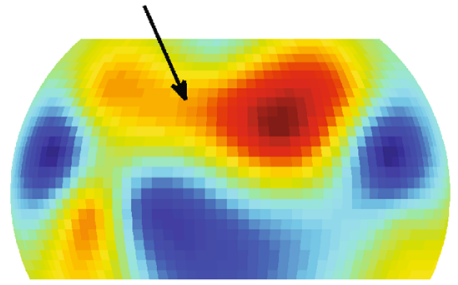

(a)

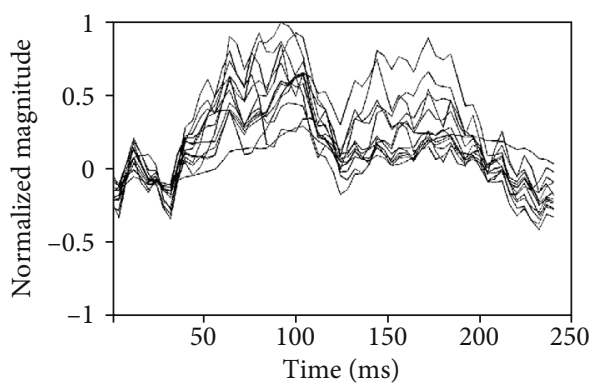

(c)

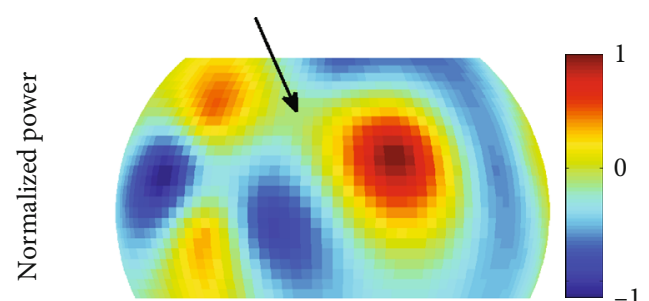

(b)

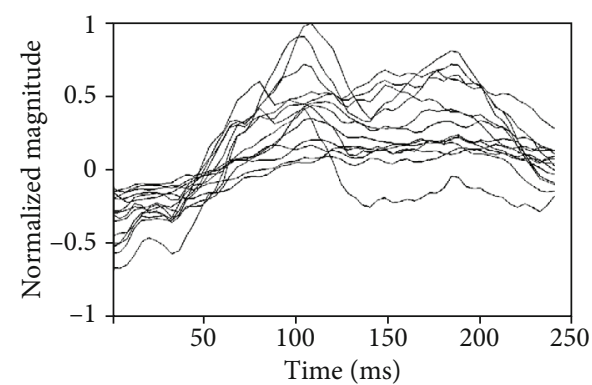

(d)

FIGURE 5: (a) Spline Laplacian VEP map (95 ms), (b) tripolar Laplacian VEP map (110 ms), (c) the normalized grand-averaged EEG VEP signals from each channel, and (d) the normalized grand-averaged tEEG VEP signals from each channel.

would be of limited value since all of the factor levels except for the two levels of factor $\mathrm{C}$ involving stochastic WGN are deterministic in nature so replicating the simulation for majority of level combinations would have yielded identical results. For the same reason, randomization of the simulation run order would have also been of limited value in our case even though in other cases it may help balancing out the effect of nuisance factors [18]. Other assumptions of ANOVA including normality, homogeneity of variance, and independence of observations were confirmed ensuring the validity of the analysis with no studentized residuals being outliers, i.e., falling outside the $[-3,3]$ range $[18]$.

In the simulation, the eccentricities of signal dipoles were set at around 0.9, closer to the surface of the brain. This alteration was made since we were mainly interested in the visual cortex area of the brain. In a previous study [24], the eccentricities of the dipoles were usually set at 0.85 or smaller. The eccentricity of the dipole has considerable impact on the Laplacian estimation. Generally, smaller eccentricities improve the performance for both spline and tripolar Laplacian estimations.

The VEP experiments showed that we can acquire VEP signals from humans and, according to the map of Figure 5(b), were able to show two separate positive regions in the TCRE Laplacian maps that were not separated in the spline Laplacian maps (Figure 5). It should be noted that we are not certain where the sources are in the visual cortex. Panels (a) and (b) are representative of the other subjects, where there were distinct positive regions in the TCRE Laplacian maps but not in the spline Laplacian maps.

Directions of future work include moving to a more realistic head model, assessing other standard EEG responses (for example, P300), and comparing how the sensitivity profile maps on the cortical surface for TCREs and conventional disc electrodes.

\section{Conclusion}

In this study, computer simulation results serve as an analytical basis for the human visual evoked potential results using half-sensitivity volume, common spatial subspace decomposition, and a comprehensive comparison between global spline surface Laplacian and local surface Laplacian estimates via tripolar concentric ring electrodes on four-layer spherical head model using full factorial design of analysis of variance. Both computer simulations and human visual evoked potential experiments suggest that there is a statistically significant improvement in spatial resolution and estimation of the Laplacian via tripolar concentric ring electrodes compared to conventional disc electrodes and the spline Laplacian but further investigation is needed for conclusive proof.

\section{Data Availability}

Part of the data used to support the findings of this study are available from the corresponding author upon request. The rest was lost due to a hardware failure after the manuscript was finalized.

\section{Disclosure}

The content is solely the responsibility of the authors and does not necessarily represent the official views of the National Science Foundation.

\section{Conflicts of Interest}

The authors declare no conflict of interest. 


\section{Acknowledgments}

We would like to thank all of our participants for their time and Zhenghan Zhu for developing the preamplifiers used to record the human VEP data. We would also like to acknowledge a partial overlap between this manuscript and Ph.D. thesis "A comparison of tripolar concentric ring electrodes to disc electrodes and an EEG real-time seizure detector design" by Xiang Liu. Parts of this research were supported by the National Science Foundation (award numbers 0933596 and 1157882 to Walter Besio as well as by award numbers 1622481 and 1914787 to Oleksandr Makeyev).

\section{References}

[1] P. L. Nunez, R. B. Silberstein, P. J. Cadusch, R. S. Wijesinghe, A. F. Westdorp, and R. Srinivasan, "A theoretical and experimental study of high resolution EEG based on surface Laplacians and cortical imaging," Electroencephalography and Clinical Neurophysiology, vol. 90, no. 1, pp. 40-57, 1994.

[2] B. He, "Brain electric source imaging: scalp Laplacian mapping and cortical imaging," Critical Reviews in Biomedical Engineering, vol. 27, no. 3-5, pp. 149-188, 1998.

[3] P. L. Nunez and R. Srinivasan, Electric Fields of the Brain: the Neurophysics of EEG, Oxford University Press, 2006.

[4] F. Babiloni, C. Babiloni, F. Carducci, L. Fattorini, P. Onorati, and A. Urbano, "Spline Laplacian estimate of EEG potentials over a realistic magnetic resonance-constructed scalp surface model," Electroencephalography and Clinical Neurophysiology, vol. 98, no. 4, pp. 363-373, 1996.

[5] C. G. Carvalhaes and P. Suppes, "A spline framework for estimating the EEG surface Laplacian using the Euclidean metric," Neural Computation, vol. 23, no. 11, pp. 2974-3000, 2011.

[6] F. Perrin, J. Pernier, O. Bertrand, and J. F. Echallier, "Spherical splines for scalp potential and current density mapping," Electroencephalography and Clinical Neurophysiology, vol. 72, no. 2, pp. 184-187, 1989.

[7] J. Le, V. Menon, and A. Gevins, "Local estimate of surface Laplacian derivation on a realistically shaped scalp surface and its performance on noisy data," Electroencephalography and Clinical Neurophysiology/Evoked Potentials Section, vol. 92, no. 5, pp. 433-441, 1994.

[8] B. Hjorth, "An on-line transformation of EEG scalp potentials into orthogonal source derivations," Electroencephalography and Clinical Neurophysiology, vol. 39, no. 5, pp. 526-530, 1975.

[9] W. G. Besio, K. Koka, R. Aakula, and W. Dai, “Tri-polar concentric ring electrode development for Laplacian electroencephalography," IEEE Transactions on Biomedical Engineering, vol. 53, no. 5, pp. 926-933, 2006.

[10] B. N. Cuffin and D. Cohen, "Comparison of the magnetoencephalogram and electroencephalogram," Electroencephalography and Clinical Neurophysiology, vol. 47, no. 2, pp. 132-146, 1979.

[11] C. Tandonnet, B. Boris, T. Hasbroucq, and F. Vidal, "Spatial enhancement of EEG traces by surface Laplacian estimation: comparison between local and global methods," Clinical Neurophysiology, vol. 116, no. 1, pp. 18-24, 2005.

[12] P. C. Hansen, "The truncatedSVD as a method for regularization," BIT Numerical Mathematics, vol. 27, no. 4, pp. 534-553, 1987.
[13] J. Malmivuo, V. Suihko, and H. Eskola, "Sensitivity distributions of EEG and MEG measurements," IEEE Transactions on Biomedical Engineering, vol. 44, no. 3, pp. 196-208, 1997.

[14] Y. Wang, P. Berg, and M. Scherg, "Common spatial subspace decomposition applied to analysis of brain responses under multiple task conditions: a simulation study," Clinical Neurophysiology, vol. 110, no. 4, pp. 604-614, 1999.

[15] A. R. Spitzer, L. G. Cohen, J. Fabrikant, and M. Hallett, “A method for determining optimal interelectrode spacing for cerebral topographic mapping," Electroencephalography and Clinical Neurophysiology, vol. 72, no. 4, pp. 355-361, 1989.

[16] R. Srinivasan, P. L. Nunez, and R. B. Silberstein, "Spatial filtering and neocortical dynamics: estimates of EEG coherence," IEEE Transactions on Biomedical Engineering, vol. 45, no. 7, pp. 814-826, 1998.

[17] R. Srinivasan, D. M. Tucker, and M. Murias, "Estimating the spatial Nyquist of the human EEG," Behavior Research Methods, Instruments, \& Computers, vol. 30, no. 1, pp. 8-19, 1998.

[18] D. C. Montgomery, Design and Analysis of Experiments, John Wiley \& Sons, 2008.

[19] R. Oostenveld and P. Praamstra, "The five percent electrode system for high-resolution EEG and ERP measurements," Clinical Neurophysiology, vol. 112, no. 4, pp. 713-719, 2001.

[20] B. He, D. Yao, J. Lian, and D. Wu, “An equivalent current source model and Laplacian weighted minimum norm current estimates of brain electrical activity," IEEE Transactions on Biomedical Engineering, vol. 49, no. 4, pp. 277-288, 2002.

[21] O. Makeyev, Y. Boudria, Z. Zhu, T. Lennon, and W. G. Besio, "Emulating conventional disc electrode with the outer ring of the tripolar concentric ring electrode in phantom and human electroencephalogram data," in 2013 IEEE Signal Processing in Medicine and Biology Symposium (SPMB), Brooklyn, NY, USA, December 2013.

[22] O. Makeyev, T. Lennon, Y. Boudria, Z. Zhu, and W. G. Besio, "Frequency domain synchrony between signals from the conventional disc electrode and the outer ring of the tripolar concentric ring electrode in human electroencephalogram data," 2014 40th Annual Northeast Bioengineering Conference (NEBEC), 2014, Boston, MA, USA, April 2014, 2014.

[23] Z. Zhu, J. Brooks, O. Makevey, S. M. Kay, and W. G. Besio, "Equivalency between emulated disc electrodes and conventional disc electrode human electroencephalography," 2014 36th Annual International Conference of the IEEE Engineering in Medicine and Biology Society, 2014, Chicago, IL, USA, August 2014, 2014.

[24] B. He, Y. Wang, and D. Wu, "Estimating cortical potentials from scalp EEGs in a realistically shaped inhomogeneous head model by means of the boundary element method," IEEE Transactions on Biomedical Engineering, vol. 46, no. 10, pp. 1264-1268, 1999. 